\title{
Malignant cerebral infarction, systemic venous thrombosis and thrombocytopenia after ChAdOx1 nCov vaccination: a possible catastrophic variant of vaccine induced thrombotic thrombocytopenia
}

Manuela De Michele ( $\square$ M.DeMichele@policlinicoumberto1.it)

Sapienza University of Rome https://orcid.org/0000-0002-3928-4309

Marta lacobucci

Sapienza University of Rome https://orcid.org/0000-0002-3911-3278

Ettore Nicolini

Sapienza University of Rome

Antonio Chistolini

Sapienza University of Rome

Fabio Pulcinelli

Sapienza University of Rome

Bruna Cerbelli

Sapienza University of Rome

Elisabetta Merenda

Sapienza University of Rome

Oscar Gaetano Schiavo

Sapienza University of Rome

\section{Emilia Sbardella}

Sapienza University of Rome

Irene Berto

Sapienza University of Rome

\section{Luca Petraglia}

Sapienza University of Rome

Nicoletta Caracciolo

Sapienza University of Rome

Massimo Chiara

Sapienza University of Rome

Simona Truglia

Sapienza University of Rome

Danilo Toni

Sapienza University of Rome 


\section{Brief Communication}

Keywords: vaccine induced thrombotic thrombocytopenia, side effect, vaccine

Posted Date: May 3rd, 2021

DOl: https://doi.org/10.21203/rs.3.rs-448338/v1

License: (c) (1) This work is licensed under a Creative Commons Attribution 4.0 International License. Read Full License

Version of Record: A version of this preprint was published at Nature Communications on August 2nd, 2021. See the published version at https://doi.org/10.1038/s41467-021-25010-x. 


\section{Abstract}

Vaccine induced thrombotic thrombocytopenia is a new syndrome recently described in young adults within two weeks from the first dose of the ChAdOx1 nCoV-19 vaccine and characterized by cerebral venous thrombosis. We report two cases of malignant middle cerebral artery (MCA) infarct and thrombocytopenia within 10 days after vaccination with ChAdOx 1 nCoV-19. Patient 1 was a 57-year-old woman who underwent decompressive craniectomy despite two successful mechanical thrombectomies. Patient 2 was a 55-year-old woman who developed a fatal bilateral malignant MCA infarct. Both the patients had pulmonary and portal vein thrombosis and high level of antibodies to platelet factor 4polyanion complexes.

\section{Introduction}

Global vaccination against severe acute respiratory syndrome-coronarovirus-2 (Sars-cov-2) has become a priority for mitigating COVID19 pandemic. Chimpanzee Adenovirus encoding the SARS-CoV-2 Spike glycoprotein (ChAdOx1) is the Oxford-AstraZeneca vaccine authorized by the European Medicines Agency on 29 January $2021 .{ }^{1}$ Three recently published papers, ${ }^{2-4}$ reported case series of unusual and mostly devastating thrombosis, in particular cerebral venous thrombosis, associated to thrombocytopenia and high level of antibodies against PF4-heparin, developing between 5 to 15 days from first dose of ChAdOx1 nCoV-19 vaccine. A similar syndrome was also observed in a young adult woman who had received the Ad26.COV2. S vaccine (Johnson \& Johnson/Janssen). ${ }^{5}$

A vaccine-induced immune thrombotic thrombocytopenia (VITT) has been suggested to name this new clinical syndrome, in order to distinguish it from the Heparin induced thrombocytopenia (HIT). ${ }^{2}$ Pathophysiological mechanisms of post-vaccination PF4-polyanion antibodies induction are still unknown. Here we describe a possible variant of the VITT characterized by a prevalent catastrophic involvement of the arterial brain district.

\section{Case Report}

Patient 1 was a 57-year-old healthy woman with a past medical history of mild hypothyroidism, in followup care after breast cancer treated surgically in 2012. Last mammography and breast ultrasound performed 4 months earlier were normal. She presented to our Emergency Department (ED) 9 days after first dose of ChAdOx1 nCoV-19 vaccine and 1 hour after onset of sudden left hemiplegia, right gaze deviation, dysarthria and left neglect caused by right middle cerebral artery (MCA) occlusion. Since blood tests showed severe isolated thrombocytopenia $\left(44,000 \mathrm{~mm}^{3}\right)$, the patient did not receive intravenous thrombolysis and underwent successful mechanical thrombectomy after platelet transfusion. Two hours later she underwent a second successful endovascular treatment after evidence of a repeated MCA occlusion at brain Magnetic Resonance (MR) and worsening of the neurological symptoms. In both procedures thrombus was collected and analyzed. Unfortunately, $12 \mathrm{~h}$ apart the patient developed a 
malignant infarct due to re-occlusion of right MCA and decompressive craniectomy was performed on day 3 from stroke onset (Fig. 1).

Intravenous betamethasone $4 \mathrm{mg}$ b.i.d. was prescribed. Total body CT scan showed extensive pulmonary artery and portal vein thrombosis, with no evidence of underlying malignancy. A trans-thoracic echocardiogram (TTE) revealed a structurally normal heart. Transcranial color Doppler ultrasonography with bubble test was negative for right-to left shunt. Platelet count continued decreasing with a nadir of $23,000 \mathrm{~mm}^{3}$ on day 2 . Intravenous high-dose ( $1 \mathrm{gr} / \mathrm{Kg}$ ) immunoglobulins (IVIg) were administered on day 4 and 5 . Platelet count increased up to $344,000 \mathrm{~mm}^{3}$ within three days, but it restarted decreasing shortly after, reaching a platelet count of $32,000 \mathrm{~mm}^{3}$ thirteen days after the IVIg administration (Fig. 2).

Treatment with fondaparinux $2,5 \mathrm{mg}$ subcutaneously u.i.d. was started when platelet count had reached $50,000 \mathrm{~mm}^{3}$.

Given the new platelet count decrease, plasma exchange was performed on 3 consecutively days (3 I exchange with $5 \%$ albumin replacement) with a low response (Fig. 2). Blood gas analysis worsened on day 11. A control thorax-CT scan was performed which showed a widespread ground-glass attenuation suggestive of a severe acute respiratory distress syndrome. Patient is still hospitalized in a critical condition.

Patient 2 was a 55-year-old healthy woman with no preexisting conditions except for mild hypothyroidism. Seven days after receiving the first dose of ChAdOx $1 \mathrm{nCoV}-19$ vaccine she started complaining of abdominal pain and presented to a first aid on the morning of day $10^{\text {th }}$. Routine blood examinations were normal except for high level of D-Dimer $(5441 \mathrm{ng} / \mathrm{mL})$ and mild thrombocytopenia (PLT 133,000). Abdominal ultrasound was normal. In the afternoon, during her hospital stay, she experienced a transient episode of aphasia and right hemiparesis followed two hours later by generalized seizures and coma. Orotracheal intubation was performed. Brain CT scan, angio-CT and perfusion CT showed occlusion of the right internal carotid artery terminus and of the left MCA, extensive ischemic core and severe bilateral hypoperfusion, without treatable penumbra (Fig. 3).

The patient was transferred to our ED. Blood examination repeated $10 \mathrm{~h}$ later showed worsening of thrombocytopenia (PLT 97,000 $\mathrm{mm}^{3}$ ) with a further decrease on the following day (PLT 59,000 $\mathrm{mm}^{3}$ ). Treatment with IVlg (1 gr/Kg per day) and dexamethasone $40 \mathrm{mg}$ u.i.d. were started on day 1. TTE was normal. Twelve-hour post-stroke a total body CT revealed extensive portal vein thrombosis with occlusion of the left intrahepatic branches and left lower lobe subsegmental pulmonary arteries thrombosis. Brain CT scan showed bilateral malignant MCA infarct with uncal herniation (Fig. 3). Brain death was declared $24 \mathrm{~h}$ later. Autopsy was not performed due to consent denial by relatives.

\section{Methods}


Both the patients were tested for COVID-19 through a nose/throat real-time reverse transcriptasepolymerase chain reaction (rRT-PCR) test. A molecular test for COVID-19 on bronchoalveolar lavage (BAL) was also performed for patient 2.

Serum antibodies (IgG) to the spike protein of Sars-cov-2 were measured with the Liaison-CLIA - DiaSorin system on blood samples of both the patients.

Coagulation Factors VIII and XIII, total levels of VWF-Antigen (VWF:Ag) and its capability to adhere to platelet glycoprotein complex GPIb-IX-V (VWF-Ristocetin Cofactor, VWF:RCo) was dosed.

Antibodies to platelet factor 4 (PF4)/polyanion in serum were tested twice in patient 1 (on day 1 and on day 15) and once in patient 2 (on day 1), by using a commercial enzyme immunoassay (EIA) (IgG/IgA/IgM, Immucor, Lifecodes, Waukesha, WI). Functional test with high concentration heparin (100 $\mathrm{IU} / \mathrm{ml}$ ) was performed. ${ }^{6}$

\section{Clot analysis}

Clot material from patient 1 was removed from stent retriever during the first endovascular procedure and collected by thromboaspiration technique during the second procedure. Histological sections obtained from the formalin fixed-paraffin embedded material were stained with hematoxylin-eosin and phosphotungstic acid-hematoxylin. Immunohistochemistry for CD61 was also performed to highlight the platelet component.

\section{Informed consent}

Written informed consent for publication and for blood samples and clot analysis was obtained from relatives of both patients.

\section{Results}

For detailed description of laboratory findings see Table S1 in Supplementary Appendix.

\section{Laboratory testing}

D-Dimer levels were elevated in both patients at admission. Coagulation parameters were normal. No signs of hemolysis were evident.

A peripheral blood smear showed giant platelets in patient 1 and platelet anisocytosis in patient 2 .

Patient 1 developed a progressive severe normochromic normocytic anemia with nadir hemoglobin level of $5,4 \mathrm{gr} / \mathrm{dL}$ on day 4 when red blood cell transfusion was performed. Patient received a second transfusion on day 14 for a subsequent reduction of hemoglobin level (nadir 7,4 gr/dL). 
Screening for thrombophilia was negative in both patients. Lupus anticoagulant, anticardiolipin and beta2 glycoprotein antibodies and antinuclear antibodies tested negative.

Coagulation Factor VIII was found increased in both the patients. Factor XIII was markedly decreased in patient 1 and not dosed in patient 2. We observed increased circulating levels of VWF:Ag and an increase in the levels of VWF:RCo.

\section{Platelet testing}

Serum of both patients showed high levels of pan antibodies (IgG, IgM and IgA) to PF4-polyanion complexes. However, patient 1 was initially not positive but high levels of antibodies were found at day 15 (>1 optical density - $\left.\mathrm{OD}_{405}\right)$. Patient 2 resulted positive since day $1\left(>1 \mathrm{OD}_{405}\right)$. The two patients did not receive heparin.

Functional activity test showed that platelets from healthy donors were clearly activated from patient 1 and 2 sera in the absence of added heparin (saline), with a percent of ATP release after 20 minutes of $22 \%$ and $14 \%$ respectively. Platelet activation was not inhibited efficiently by high-dose heparin in both the patients (percent of ATP release after 20 minutes of $19 \%$ and $9 \%$ respectively). Serum from healthy volunteers did not induce platelet secretion.

\section{COVID-19 serological testing.}

Serum antibodies to spike protein of Sars-CoV-2 (IgG) were positive in patient 1 and negative in patient 2. Both the patients had negative COVID-19 rRT-PCR test on nasopharyngeal swab. Patient 2 BAL tested on day 1 was also negative.

\section{Clot analysis}

Clot collected from patient 1 (Fig.S1) during the first thrombectomy was mainly composed of - platelets ( $85 \%$ of the total material examined) and was massively infiltrated by neutrophils with scarce evidence of karyorrhexis. Histological features consistent with the presence of neutrophil DNA Extracellular Traps (NETs) were observed.

Clot collected during the second endovascular procedure was a red blood cell-rich thrombus $(90 \%$ of red blood cells and $10 \%$ fibrin and platelets) with scarce neutrophils (not shown).

The data of this report are available from the corresponding author, upon reasonable request.

\section{Discussion}

We present two cases of malignant cerebral infarct, systemic venous thrombosis and concomitant thrombocytopenia in two young healthy adult women within 10 days from vaccination against SARS- 
CoV-2 with ChAdOx1 nCov-19. High serum levels of antibodies to PF4-polyanion complexes were found in both patients.

Some relevant findings from these two cases need considerations. Patient 1 had a right MCA stroke due to occlusion of the M1 segment. Despite two successful endovascular treatments, the patient experienced a repeated occlusion of the same vessel and developed a malignant MCA infarct. This unusual event suggests a high prothrombotic status and local endothelium dysfunction. According to this assumption, histology of the first removed thrombus showed a platelet-rich clot with neutrophils. Platelet-rich thrombi are formed by VWF, NETs and fibrin. ${ }^{7}$ Platelet/fibrin thrombi were also found in experimental model of HIT in veins and arteries of multiple organs. ${ }^{8}$ Pathogenesis of the severe normochromic normocytic anemia developed by patient 1 has not yet been elucidated and it is still under investigation. We found high level of Sars-cov-2 IgG serum antibodies suggestive of a previously COVID19 asymptomatic infection. No Sars-CoV-2 IgG serum antibodies were present in serum of patient 2 . This data would suggest that anti-spike protein antibodies may not be relevant in the pathogenesis of this syndrome. Interestingly, antibodies to PF4-polyanion complexes were initially negative in serum of patient 1 , while high antibody level was found in serum collected and tested two weeks later.

The serologic pattern of these two cases is similar to that of the so called autoimmune HIT, since serum from our patients strongly activated platelets in the absence of heparin (heparin-independent platelet activation). ${ }^{9}$ Moreover, platelet secretion was scarcely sensitive to inhibition with high-dose heparin, as observed in two patients described by Schultz et al. ${ }^{3}$ and in one described by Greinacher et al. ${ }^{2} \mathrm{~A}$ disseminated intravascular coagulation was not observed in our patients.

Both the patients had extensive arterial thrombosis of the lung and venous thrombosis of the splanchnic district. An hypercoagulable state was observed in both the patients with high level of VWF and VWF:RCo, Ddimer, and coagulation Factor VIII. Factor XIII was markedly decreased in patient 1, probably due to excessive consumption within thrombi. ${ }^{10}$

Treatment of this new syndrome is challenging. Administration of high-dose IVlg has been suggested in attempt to inhibit Fcy receptor-mediated platelet activation, since in the treatment of severe autoimmune HIT this treatment resulted in rapid increase in platelet count. ${ }^{9,11}$ Unfortunately, platelet response of patient 1 to high dose IVIG was striking but transient. Poor platelet increase was observed after three sessions of PEx.

Many scientific questions regarding VITT are still unanswered as highlighted by a recently published editorial. ${ }^{12}$ Although limited to two patients, data collected in this report strengthen the hypothesis that platelets could be initially activated by unknown factors related to the ChAdOx1 nCov-19 vaccine /host interaction. An atypical autoimmune HIT may be the consequent event which could amplify platelet activation and coagulation cascade. The rare occurrence of VITT also in patients who received the Ad26.COV2.S vaccine (Johnson \& Johnson/Janssen) ${ }^{5}$ suggests a possible pathogenic role of the adenoviral viral vector. More investigations are warranted to elucidate this hypothesis. 
In conclusion, our two cases of young adult women with massive brain artery thrombosis in addition to extensive systemic venous thrombosis, thrombocytopenia and PF4-polyanion antibodies, developed within 10 days from ChAdOx $1 \mathrm{nCov}-19$ vaccination, might represent a stroke variant of the recently named VITT syndrome. More investigations are urgently needed in order to better understand the pathogenesis of this catastrophic syndrome.

\section{Declarations}

\section{Informed consent}

Written informed consent for publication and for blood samples and clot analysis was obtained from relatives of both patients.

\section{References}

1. Voysey M, Clemens SAC, Madhi SA, et al. Safety and efficacy of the ChAdOx1 nCoV-19 vaccine (AZD1222) against SARS-CoV-2: an interim analysis of four randomised controlled trials in Brazil, South Africa, and the UK. Lancet 2021;397(10269):99-111.

2. Greinacher A, Thiele T, Warkentin TE, Weisser K, Kyrle PA, Eichinger S. Thrombotic Thrombocytopenia after ChAdOx1 nCov-19 Vaccination. N Engl J Med [Internet] 2021;NEJMoa2104840. Available from: http://www.ncbi.nlm.nih.gov/pubmed/33835769

3. Schultz NH, Sørvoll IH, Michelsen AE, et al. Thrombosis and Thrombocytopenia after ChAdOx1 nCoV19 Vaccination. N Engl J Med [Internet] 2021;(Table 1):NEJMoa2104882. Available from: http://www.ncbi.nlm.nih.gov/pubmed/33835768

4. Scully M, Singh D, Lown R, et al. Pathologic Antibodies to Platelet Factor 4 after ChAdOx1 nCoV-19 Vaccination. N Engl J Med [Internet] 2021;NEJMoa2105385. Available from: http://www.ncbi.nlm.nih.gov/pubmed/33861525

5. Muir K-L, Kallam A, Koepsell SA, Gundabolu K. Thrombotic Thrombocytopenia after Ad26.COV2.S Vaccination. N Engl J Med [Internet] 2021;Correspond(1):NEJMc2105869. Available from: https://www.nejm.org/doi/full/10.1056/NEJMc2105869

6. Guarino ML, Massimi I, Mardente S, et al. New platelet functional method for identification of pathogenic antibodies in HIT patients. Platelets [Internet] 2017;28(7):728-30. Available from: https://doi.org/10.1080/09537104.2017.1293803

7. Staessens $S$, Denorme $F$, Francois 0 , et al. Structural analysis of ischemic stroke thrombi: histological indications for therapy resistance. Haematologica [Internet] 2020;105(2):498-507. Available from: http://www.haematologica.org/lookup/doi/10.3324/haematol.2019.219881

8. Reilly MP, Taylor SM, Hartman NK, et al. Heparin-induced thrombocytopenia/thrombosis in a transgenic mouse model requires human platelet factor 4 and platelet activation through FcyRIIA. Blood [Internet] 2001;98(8):2442-7. Available from: http://dx.doi.org/10.1182/blood.V98.8.2442 
9. Greinacher A, Selleng K, Warkentin TE. Autoimmune heparin-induced thrombocytopenia. J Thromb Haemost [Internet] 2017;15(11):2099-114. Available from: http://doi.wiley.com/10.1111/jth.13813

10. Ząbczyk M, Natorska J, Undas A. Factor XIII and Fibrin Clot Properties in Acute Venous

Thromboembolism. Int J Mol Sci [Internet] 2021;22(4):1607. Available from:

https://www.mdpi.com/1422-0067/22/4/1607

11. Warkentin TE. High-dose intravenous immunoglobulin for the treatment and prevention of heparininduced thrombocytopenia: a review. Expert Rev Hematol [Internet] 2019;12(8):685-98. Available from: https://doi.org/10.1080/17474086.2019.1636645

12. Cines DB, Bussel JB. SARS-CoV-2 Vaccine-Induced Immune Thrombotic Thrombocytopenia. N Engl J Med [Internet] 2021;NEJMe2106315. Available from:

http://www.nejm.org/doi/10.1056/NEJMe2106315

\section{Figures}

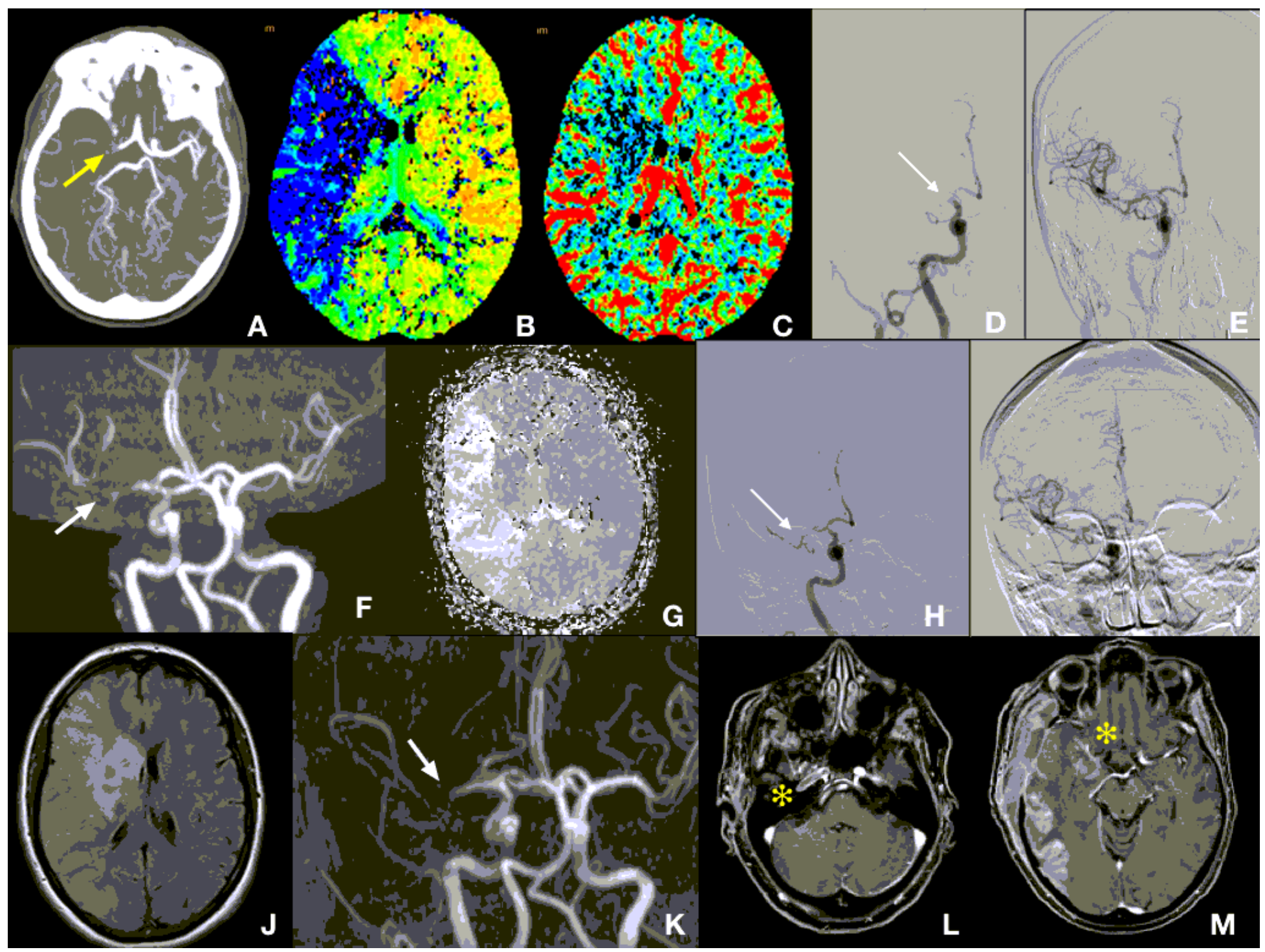

Figure 1 
Patient 1. A: Computed Tomograghy Angiography (CTA) showed proximal M1 segment occlusion of right middle cerebral artery (MCA) (yellow arrow); B and C: CT perfusion (CTP) maps: mean transit time (B) and cerebral blood volume (CBV) (C) showed a large area of mismatch indicating salvageable penumbra; $D$ an E: Endovascular mechanical thrombectomy achieved completed recanalization (E) of the MCA occlusion (white arrow in D); F and G: MCA reocclusion on M2 segment 2 hours after the procedure, as showed by 3D-time of flight (TOF) magnetic resonance imaging (MRI) sequence (white arrow in F), with ischemic penumbra on mean transit time map in perfusion MRI (G); $\mathrm{H}$ and $\mathrm{I}$ : Second complete endovascular recanalization ( $\mathrm{H}$ before and I after the mechanical thrombectomy); $\mathrm{J}$ and $\mathrm{K}$ : Fluid attenuated inversion recovery MRI at 12 hours showed the extension of ischemia to superficial and deep right MCA territory with mass effect on the ventricular system $(\mathrm{J})$, with occlusion of MCA at TOF Magnetic Resonance Angiography (white arrow in K). Extension of the arterial occlusion to the right internal carotid artery (yellow asterisk in $L$ and $M$ ).

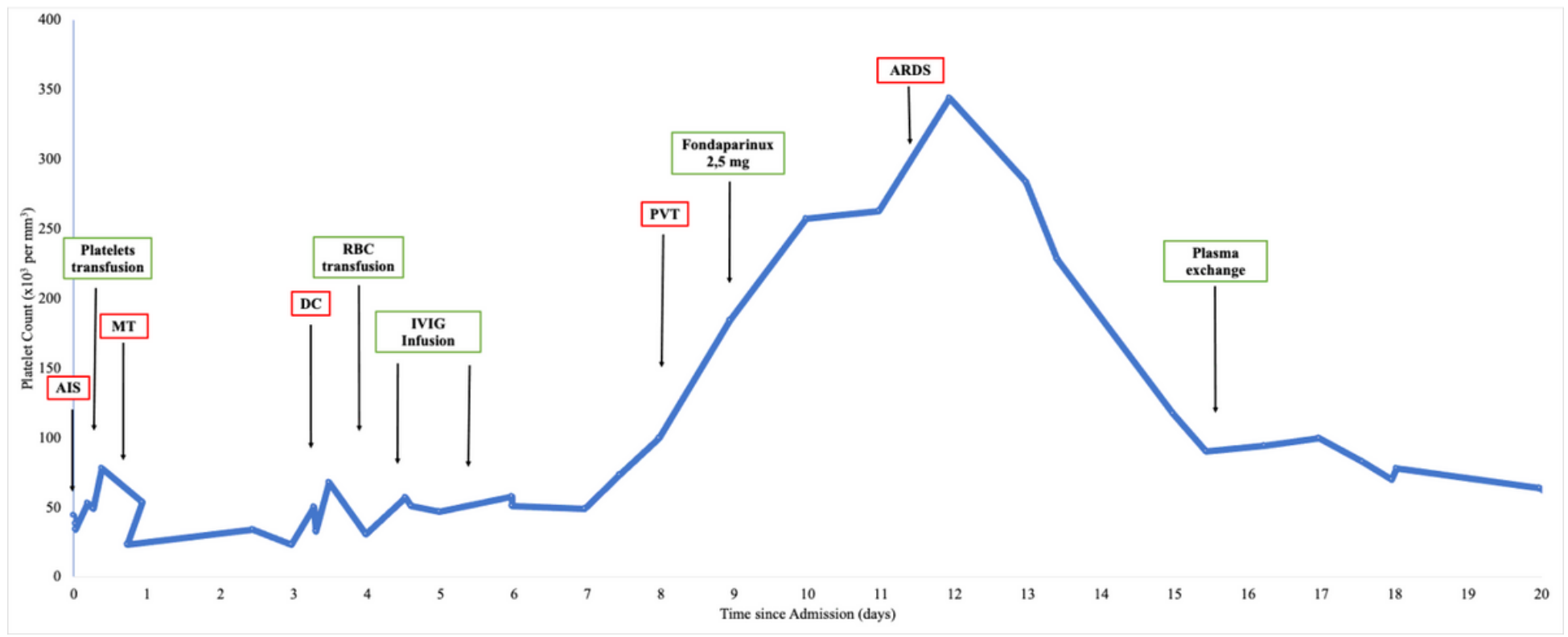

\section{Figure 2}

Platelet count course in Patient 1. AIS= acute ischemic stroke; $M T=$ mechanical thrombectomy; $D C=$ decompressive craniectomy; $\mathrm{PVT}=$ portal vein thrombosis; $\mathrm{ARDS}=$ acute respiratory distress syndrome. 


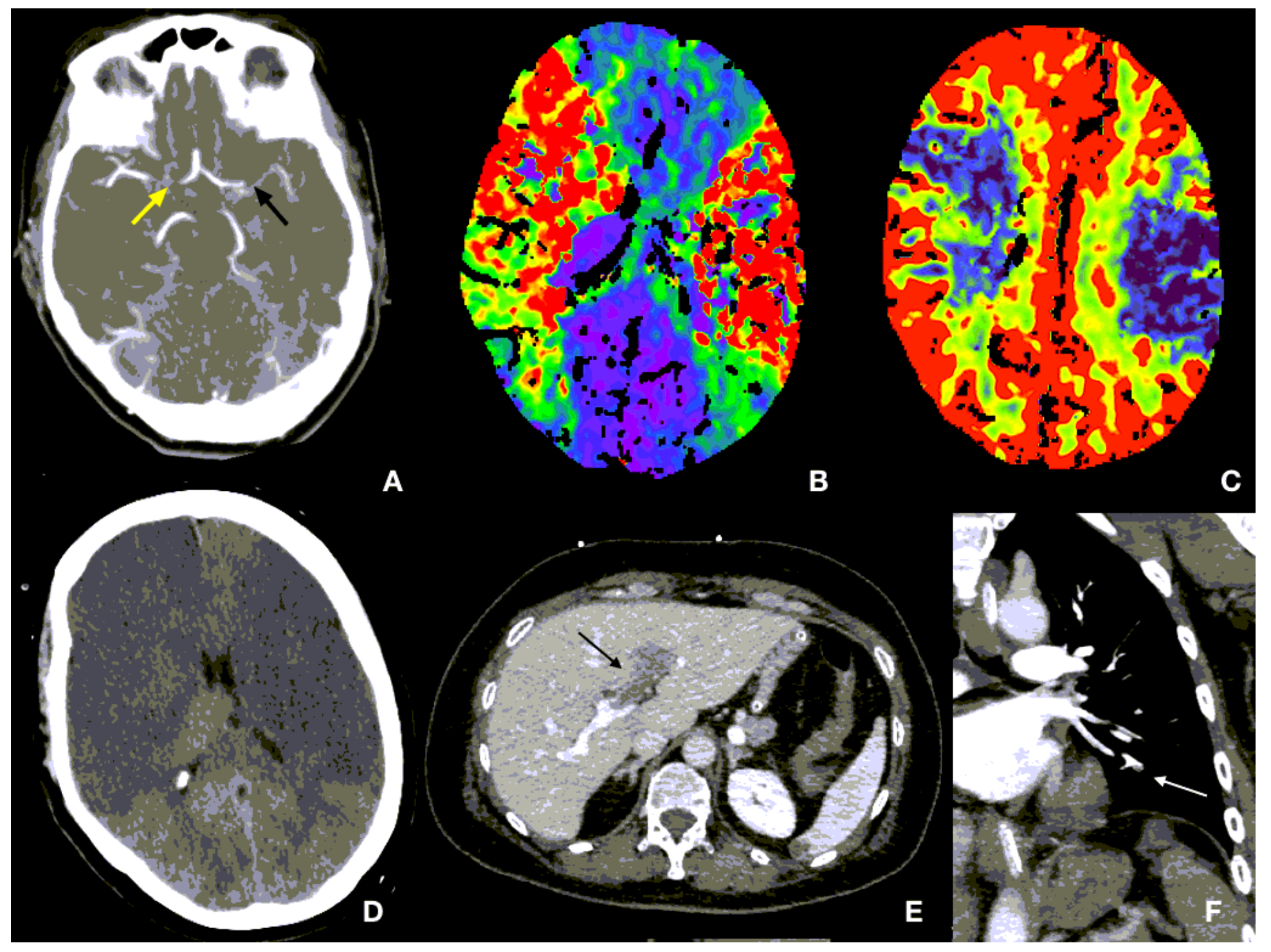

Figure 3

Patient 2. A: CTA showed the proximal M1 segment occlusion of left middle cerebral artery (black arrow) and the occlusion of the right internal carotid artery terminus (yellow arrow); B: CTP showed bilateral infarct core (CBV map in C) and hypoperfusion (Mean Transit Time - MTT map in B), without treatable penumbra; D: 24 hours after, brain CT revealed bilateral malignant MCA infarcts; E: portal vein thrombosis with extension to the left intrahepatic branches (black arrow); F: subsegmental pulmonary artery thrombosis (white arrow).

\section{Supplementary Files}

This is a list of supplementary files associated with this preprint. Click to download.

- SupplementaryAppendix.docx

- flatBC113054epc.pdf

- flatBC113054rs.pdf 